\title{
The low dimensional dynamical system approach in General Relativity: an example
}

\author{
H. P. de Oliveira and E. L. Rodrigued \\ Instituto de Física, Universidade do Estado do Rio de Janeiro \\ R. São Francisco Xavier, 524, CEP 20550-013 \\ Rio de Janeiro, RJ, Brazil \\ I. Damião Soarest \\ Centro Brasileiro de Pesquisas Físicas/MCT \\ R. Dr. Xavier Sigaud, 150, CEP 22290-180 \\ Rio de Janeiro, RJ, Brazil \\ E. V. Tonin: 3 \\ Centro Federal de Educação Tecnológica do Espírito Santo \\ Avenida Vitória, 1729, CEP 29040-780 \\ Vitória, ES, Brazil \\ (Dated: July 14, 2018)
}

\begin{abstract}
In this paper we explore one of the most important features of the Galerkin method, which is to achieve high accuracy with a relatively modest computational effort, in the dynamics of RobinsonTrautman spacetimes.

PACS numbers:
\end{abstract}

\section{INTRODUCTION}

The General Theory of Relativity (GR) constitutes one of the major scientific achievements of the past hundred years. Its theoretical framework establishes that the gravitational field and the geometry of the spacetime are the same physical entity and that the equations ruling the gravitational dynamics are field equations of the spacetime geometry. GR has so far passed extremely well all experimental tests [1], in opposition to other relativistic extensions as scalar-tensor theories. Basically, GR establishes a system of ten nonlinear, coupled partial differential equations that governs the dynamics of the gravitational field represented by the symmetric second order metric tensor $g_{\alpha \beta}, \alpha, \beta=0,1,2,3$. The nonlinear nature of the field equations is the main obstacle to obtain exact solutions of the field equations, unless we make appeal to symmetries in the solutions, or alternatively the use of perturbative approaches are adopted. Therefore, in order to study the dynamics of the gravitational field in more general situations the use of numerical techniques seems to be the only possible strategy to circumvent the difficulty posed by the nonlinearities of the field equations. In this context numerical rel-

\footnotetext{
*Electronic address: oliveira@dft.if.uerj.br

$\dagger$ Electronic address: elrodrigues@uerj.br

${ }^{\ddagger}$ Electronic address: ivano@cbpf.br

$\S$ Electronic address: tonini@cefetes.br
}

ativity has become a very fertile and at the same time challenging field of research as recently considered in several interesting reviews [2]. There the improvement of specific numerical techniques adapted to relativistic problems, along with the increase of computational resources figure as promising factors for the advance of numerical relativity. Nonetheless, despite all the progress made so far the complete understanding of important problems in relativistic astrophysics such as non-spherical collapse and nonlinear regimes of emission of gravitational waves is still not complete.

A promising approach to treat numerically nonlinear problems is provided by the so-called spectral methods [3]. The spectral methods adopt a distinct strategy if compared with the finite difference scheme. For instance, considering a function $u(t, x)$ satisfying a given one dimensional partial differential equation, it will be approximate as a series of the type $u_{a}(t, x)=\sum_{k=0}^{N} a_{k}(t) \psi_{k}(t)$, where the basis or trial functions $\psi_{k}(x)$ are known analytical polynomials such as Fourier, Legendre, Chebyshev, etc. In general, by increasing the truncation order $N, u_{a}(t, x)$ approaches of the exact solution of the problem in the mean. There are distinct types of spectral methods among which we list the Galerkin method [4, [5], the collocation method [] $]$ and the Taumethod. These methods have an attractive feature which is to transform any partial differential equation into a finite set of coupled ordinary differential equations, or simply a dynamical system whose dimension is dictated by the truncation order $N$. An- 
other important and robust feature is the high accuracy achieved with a small truncation order, what corresponds to use moderate or low computational resources.

In this paper our objective is to present a consistent low dimensional dynamical system approach provided by the Galerkin method applied to the problem of non-spherical collapse with the emission of gravitational waves, in the realm of RobinsonTrautman geometries [7]. In this way, the paper is divided as follows. The basic equations that govern the dynamics of Robinson-Trautman spacetimes are presented in Section 2. In Section 3 we discuss and implement efficiently the Galerkin method. In Section 4 numerical experiments are performed in order to test the efficiency and convergence of the method. Section 5 is devoted to the conclusions and final remarks.

\section{THE DYNAMICS OF ROBINSON-TRAUTMAN SPACETIMES: THE NONLINEAR DIFFERENTIAL EQUATION}

Robinson-Trautman (RT) spacetimes are the simplest axisymmetric spacetimes that can be viewed as the exterior geometry of an isolated source emitting gravitational waves [7, 8] or the exterior of a distorted black hole. In a suitable coordinate system the line element for RT geometries is expressed as

$$
\begin{aligned}
d s^{2}= & \left(\lambda(u, \theta)-\frac{2 m_{0}}{r}+2 r \frac{\dot{K}(u, \theta)}{K(u, \theta)}\right) d u^{2}+2 d u d r- \\
& r^{2} K^{2}(u, \theta)\left(d \theta^{2}+\sin ^{2} \theta d \varphi^{2}\right),
\end{aligned}
$$

where $m_{0}$ is a constant related to the total mass of the system and dot means derivative with respect to $u$. According to Einstein field equations the function $\lambda(u, \theta)$ is related to $K=K(u, \theta)$ by the constraint equation

$$
\lambda(u, \theta)=\frac{1}{K^{2}}-\frac{K_{\theta \theta}}{K^{3}}+\frac{K_{\theta}^{2}}{K^{4}}-\frac{K_{\theta}}{K^{3}} \cot \theta,
$$

where the subscript $\theta$ denotes derivative with respect to the angle $\theta$. The remaining vacuum Einstein equations impose that the function $K(u, \theta)$ satisfies the evolution equation

$$
-6 m_{0} \frac{\dot{K}}{K}+\frac{\left(\lambda_{\theta} \sin \theta\right)_{\theta}}{2 K^{2} \sin \theta}=0,
$$

which henceforth will be denoted as the RobinsonTrautman (RT) equation. This equation governs the evolution of the gravitational field, in other words, it allows to evolve the initial data $K_{0}(\theta)=$ $K\left(u=u_{0}, \theta\right)$ prescribed on a given null surface $u=u_{0}=$ constant (except in the case $m_{0}=0$ ). Despite its relatively simple form no general exact non-stationary solution of RT equation is known. However by restricting to stationary configurations two important solutions are known. The first is the Schwarzschild solution that represents the gravitational field a black hole characterized by $K=K_{0}=$ constant, which implies $\lambda=K_{0}^{-2}$ with a corresponding Schwarzschild mass $M=m_{0} K_{0}^{3}$. The second solution represents a boosted black hole [9] along the axis of symmetry such that

$$
K(\theta)=\frac{1}{\cosh \gamma+\cos \theta \sinh \gamma}
$$

where $\gamma$ is a parameter associated with the velocity of the boost, $v=\tanh \gamma$. The constraint equation (2) in this case yields $\lambda=1$.

\section{THE SPECTRAL METHOD APPROACH}

It will be useful to express $K(u, \theta)$ as

$$
K(u, x)=A_{0} e^{\frac{1}{2} Q(u, x)},
$$

where $A_{0}$ is a constant, and for convenience we have introduced the variable $x=\cos \theta$ with $-1 \leq x \leq 1$. The first step to apply the Galerkin method is to express $Q(u, x)$ as a power series with respect to a suitable set of basis functions chosen as the Legendre polynomials due to the symmetry of the problem and the required regularity of $K(u, x)$ with respect to $x$. In this way an approximated expression for $Q(u, x)$ is established and given by

$$
Q_{a}(u, x)=\sum_{k=0}^{N} b_{k}(u) P_{k}(x)
$$

where $N$ is the truncation order, $b_{k}(u)$ and $P_{k}(x)$ are the modal coefficients and the Legendre polynomials of kth order. This basis functions define an abstract projection space for which an internal product can be defined, in this case, as

$$
\left\langle P_{j}(x), P_{k}(x)\right\rangle=\int_{-1}^{+1} P_{j}(x) P_{k}(x) d x=\frac{2 \delta_{k j}}{2 k+1} .
$$

The next step is to substitute the Galerkin decomposition for $Q(u, x)$ into Eq. (2) to obtain an approximated expression for $\lambda(u, x)$, or 
$\lambda_{a}(u, x)=\frac{e^{-Q_{a}(u, x)}}{A_{0}^{2}}\left(1+\sum_{k=0}^{N} \frac{1}{2} k(k+1) b_{k}(u) P_{k}(x)\right)$.

The so called residual equation follows after introducing the approximated expressions for $Q(u, x)$ and $\lambda(u, x)$ given above into the RT equation (3). After some direct calculation we obtain schematically

$\operatorname{Res}(u, x)=\sum_{k=0}^{N} b_{k}(u) P_{k}(x)-\frac{\mathrm{e}^{-Q_{a}(u, x)}}{6 m_{0} A_{0}^{2}}\left[\left(1-x^{2}\right) \lambda_{a}^{\prime}\right]^{\prime}$,

where prime stands for derivative with respect to $x$. In fact the residual equation can be a good measure of the error of truncation and a valuable test [10] for the convergence of the Galerkin decomposition. According to the traditional Galerkin method the projection of the residual equation into each basis function $P_{n}(x), n=0,1, \ldots, N$ is assumed to be zero in order to minimizing the error of truncation, or $\left\langle\operatorname{Res}(u, x), P_{n}(x)\right\rangle=0$, rendering the following dynamical system for the modal coefficients

$$
\begin{aligned}
\dot{b}_{n}(u)= & \frac{(2 n+1)}{12 m_{0} A_{0}^{2}} \int_{-1}^{1} \exp \left(-\sum_{k=0}^{N} b_{k}(u) P_{k}(x)\right) \times \\
& {\left[\left(1-x^{2}\right) \lambda_{a}^{\prime}\right]^{\prime} P_{n}(x) d x, }
\end{aligned}
$$

with $n=0,1, \ldots, N$.

Here some remarks are necessary. In order to express the rhs of the above equation in terms of the modal coefficients, it is necessary to integrate a large expression involving an exponential of a series of Legendre functions, the size of which depends on the truncation order $N$. This integration must be necessarily done symbolically (in Maple or Mathematica), but unless $N \leq 2$ - a very low truncation order such an integration cannot be performed. Thus, we can adopt two distinct strategies based on further approximation schemes:

- The first is to assume

$$
e^{-Q_{a}}=1-Q_{a}+\frac{Q_{a}^{2}}{2 !}+\ldots \cong \sum_{j=0}^{J} \frac{\left(-Q_{a}\right)^{j}}{j !},
$$

which renders a polynomial expression for the exponential. Introducing this expression into Eq (9) the integration can be done promptly without much cost of CPU time for reasonable choices of $N$ and $J$. As a matter of fact, this additional approximation can be acceptable for not large values of $|Q|$ as we are going to see later.
- The second strategy consists, on the hand, in applying the collocation method [6] to express $\mathrm{e}^{-Q_{a}(u, x)}$ as a series of another basis functions, or

$$
\mathrm{e}^{-Q_{a}(u, x)} \cong \sum_{k=0}^{M} q_{k}(u) T_{k}(x),
$$

where $T_{k}(x)$ are the Chebyshev polynomials, and $q_{k}(u)$ the new modal coefficients given as functions of the original coefficients $b_{j}(u)$. These relations are fixed by the fact that at the collocation points $x_{j}=\cos (\pi j / M)$, $j=0,1, \ldots, M$ the above decomposition agrees with the exact expression, or

$$
\mathrm{e}^{-Q_{a}\left(u, x_{j}\right)}=\sum_{k=0}^{M} q_{k}(u) T_{k}\left(x_{j}\right)
$$

and together with the discrete orthogonality relation $\delta_{j k}=\frac{2}{M \bar{c}_{k}} \sum_{n=0}^{M} \frac{1}{\bar{c}_{n}} T_{j}\left(x_{n}\right) T_{k}\left(x_{n}\right)$, where $\bar{c}_{0}=\bar{c}_{M}=2$ and $\bar{c}_{k}=1$, for $1 \leq k \leq$ $M-1$. As a consequence each modal coefficient $q_{k}$ can be expressed in terms of $b_{k}$. In both schemes a $N+1$ dimensional dynamical system for the modal coefficients is obtained after integrating the rhs of Eq. (10).

\section{NUMERICAL EXPERIMENTS}

Our first numerical task will be to study the accuracy and convergence of the approximative schemes outlined at the end of the last Section. To this end we consider the exact expressions for some initial data $K_{0}(x)=K\left(u_{0}, x\right)$ identified as an oblate spheroid and a black hole in motion given, respectively, by

$$
\begin{aligned}
K_{0}(x) & =\frac{k_{0} \sqrt{1-e^{2}}}{\sqrt{1-\left(1-x^{2}\right) e^{2}}} \\
K_{0}(x) & =\frac{1}{\cosh \gamma+x \sinh \gamma},
\end{aligned}
$$

where $k_{0}$ and $\gamma$ are constants and $e$ is the eccentricity. As a further assumption the eccentricity can depend on $x$ as $e=e_{0}+e_{j} x^{j}$, with $e_{0}, e_{j}$ being parameters, in order to encompass the usual oblate spheroid $\left(e_{j}=0\right.$ and $\left.0<e_{0}<1\right)$, and a deformed oblate spheroid $\left(e_{0}>e_{j} \neq 0\right)$. It is worth of calling attention that no dynamics is present if the second 

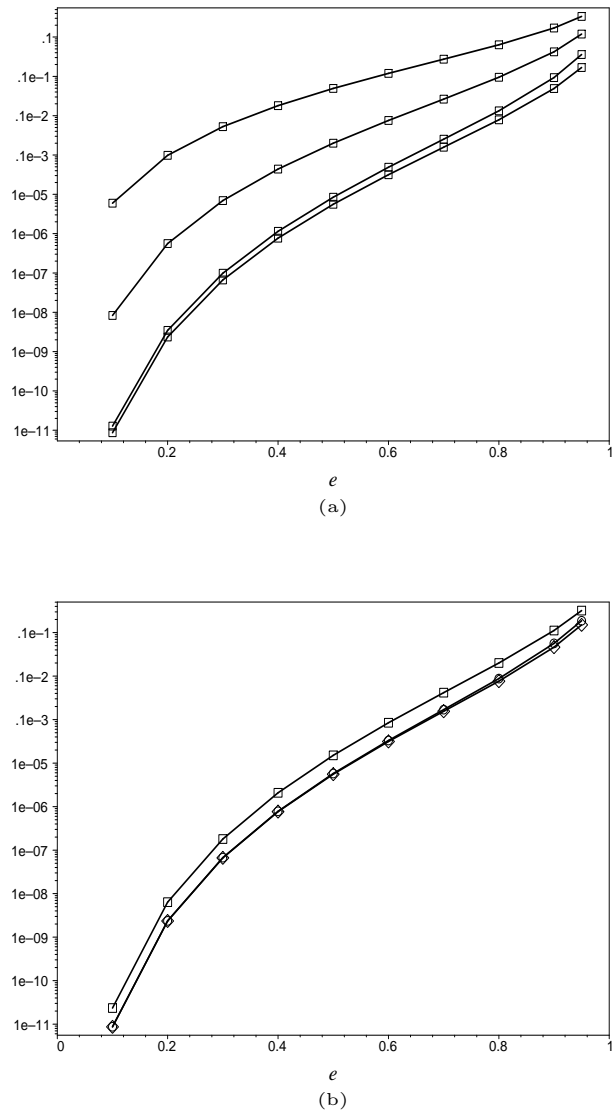

FIG. 1: $L_{2}$ error for the oblate spheroid $\left(e_{j}=0\right)$ in function of the eccentricity $e$ for $J=1,2,3,4$ (upper to lower curves). Similar plots are constructed in (b) considering the second scheme of approximation, for which the we have selected $M=6,8$ and 10 collocation points starting from the upper curve (boxes). Note that the last two curves almost coincide indicating a rapid convergence of the method, whereas in (a) the convergence is evident only if $J \geq 3$. In all cases we have set $N=7$.

data family is chosen, since it represents an exact stationary solution of the RT equation.

According to the previous discussion we choose the truncation order $N$ from which the decomposition $Q_{a}\left(u_{0}, x\right)=\sum_{k=0}^{N} b_{k}\left(u_{0}\right) P_{k}(x)$ is established. The values of each modal coefficient $b_{k}\left(u_{0}\right)$ will be determined using the standard Galerkin projection procedure starting from $K_{0}(x) \simeq K_{a}(x)=$ $A_{0} \exp \left(1 / 2 \sum_{k=0}^{N} b_{k}(u) P_{k}(x)\right)$ that yields

$$
b_{k}\left(u_{0}\right)=\frac{\left\langle 2 \ln \left(A_{0}^{-1} K_{0}(x), P_{k}(x)\right\rangle\right.}{\left\langle P_{k}(x), P_{k}(x)\right\rangle} .
$$

Henceforth, we set $A_{0}=k_{0}=1$ without loss of generality. Once the modal coefficients are determined $\exp \left(1 / 2 Q_{a}\right)$ will be further approximated according to the schemes presented in the last Section: the first is to expand the exponential as a power series of $-Q / 2$ in the same way as shown in Eq. (11); the second is to expand the exponential using the collocation method. In order to provide a quantitative measure of the error between these approximations and the exact expressions for the corresponding initial data, the $L_{2}$ error defined by

$$
L_{2}=\sqrt{\frac{1}{2} \int_{-1}^{1}\left[K_{0}(x)-K_{a}(x)\right]^{2} d x}
$$

will be evaluated in each case.
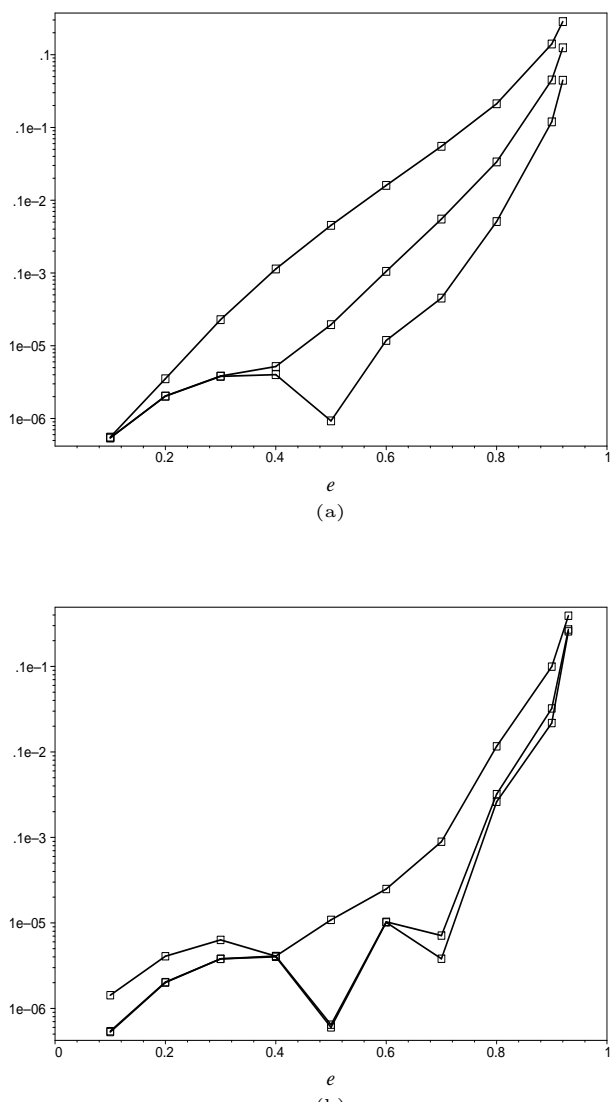

(b)

FIG. 2: $L_{2}$ error for the deformed oblate spheroid $\left(e_{2}=\right.$ 0.07 ) in function of the eccentricity $e_{0}$ for (a) $J=2,3,4$ (upper to lower curves). Similar plots are constructed in (b) considering the second scheme of approximation, for $M=6,8$ and 10 collocation points starting from the upper curve. Note that as shown in Fig. 1(b) the last two curves almost coincide indicating a rapid convergence of the method. In all cases we have set $N=7$.

Let us consider the oblate spheroid with $e_{j}=0$ and $0<e<1$. We then fix the truncation 
order to $N=7$ and evaluate the error $L_{2}$ between the exact and approximate expressions for the oblate spheroid taking into account both additional schemes as previously described. In the first we choose $J=1,2,3$ and 4 present in the expansion $\exp \left(Q_{a} / 2\right) \simeq \sum_{k=0}^{J} 2^{-k} Q_{a}^{k} / k !$, whereas for the second scheme the expansion of $\exp \left(Q_{a} / 2\right)$ is constructed with $M=6,8$ and 10 collocation points. The respective $L_{2}$ errors evaluated for successive values of the eccentricity $e$ are shown in Fig. 1. Two important aspect are worth to be mentioned. As expected small eccentricities produce very small errors even for the quite modest choice $J=1$, since these cases correspond to tiny departures from the spherically symmetric configuration described exactly by the Galerkin decomposition as $b_{0}\left(u_{0}\right)=$ constant, $b_{k}\left(u_{0}\right)=0$ for $k \neq 0$. High eccentricities, otherwise, represent more severe deviations from the spherical configuration, producing as a consequence an increase of the error. The second aspect concerns to the fast convergence achieved using both approaches as suggested by the plots of Figs. 1(a) and 1(b). Accordingly, in the first case the convergence is clear if $J \geq 3$, and it is necessary that the number of collocation points is greater than, or $N \geq 8^{1}$. Despite the acceptable errors attained with a modest truncation order $(N=7)$ in both cases, the use of the second scheme appears to be superior because the less algebraic effort necessary to achieve the same results of the first case with $J=4$.

In Fig. 2 we have evaluated the $L_{2}$ error for the (deformed) oblate spheroid with variable eccentricity $e=e_{0}+e_{2} x^{2}$, in which we have set $e_{2}=0.07$ and again $0<e<1$. As it can be seem from the plots it is clear that the scheme using the collocation points is more efficient, in the sense of the need of smaller computational effort, and accurate than the first scheme. The initial data representing a black hole in motion is considered in Fig. 3, where $J=4$ and $M=8$ collocation points which confirms once more the previous conclusion.

In the second group of numerical experiments we shall verify the evolution of the error associated to the constraint equation (2). The procedure consists into rewrite Eq. (2) in the following way

$$
\lambda K^{2}+\left[\left(1-x^{2}\right) \frac{K^{\prime}}{K}\right]^{\prime}-1=0
$$

1 The convergence depends on the truncation order, but we have noticed that for a fixed truncation order $N$ the most efficient decomposition is obtained if $M=N+1$ collocation points is selected. If $M>N+1$ there is not relevant improvement in the error as exemplified in Fig. 1(b).

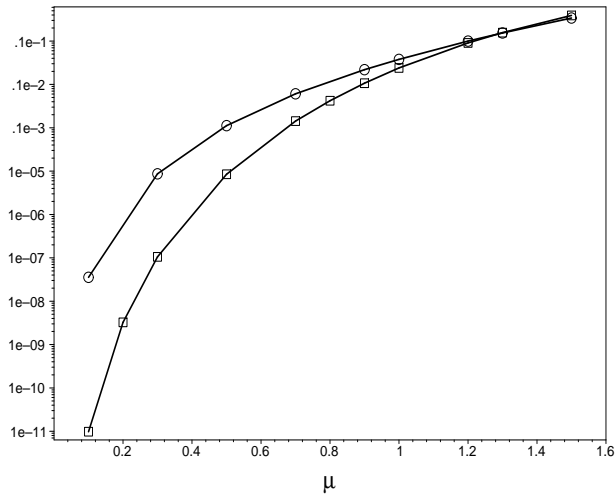

FIG. 3: $L_{2}$ error for the initial data identified as the boosted black hole (13) if the truncation order is $N=7$, and taking into account the collocation scheme $(M=8$, lower curve) and the first scheme with $J=4$ (upper curve) for the first scheme.

Here $\lambda(u, x)$ is given by Eq. (8), but $K(u, x)$ will be expressed according to the schemes of approximation as outlined by Eqs. (11) and (12). For instance, by assuming the expansion provided by the later expression we have

$$
K(u, x)=A_{0}^{2} \mathrm{e}^{Q} \simeq A_{0}^{2}\left(\left(\sum_{k=0}^{M} q_{k}(u) T_{k}(x)\right)^{-1},\right.
$$

and, as a consequence, the constraint equation now reads

$$
\begin{aligned}
& \mathrm{CE}(u, x)=\lambda_{a} A_{0}^{2}\left(\sum_{k=0}^{M} q_{k}(u) T_{k}(x)\right)^{-1} \\
& -\frac{1}{2}\left[\left(1-x^{2}\right) \frac{\sum_{k=0}^{M} q_{k}(u) T_{k}^{\prime}(x)}{\sum_{k=0}^{M} q_{k}(u) T_{k}(x)}\right]^{\prime}-1=0 .(17)
\end{aligned}
$$

A similar expression is obtained if the approximation expansion given by (11) is assumed. In any case the resulting form of the constraint equation is evaluated at each instant after the integration of the dynamical equation for the modal coefficients. As a matter of fact, we are interested in the rms error of the constraint or

$$
\sqrt{\frac{1}{2} \int_{-1}^{1} \mathrm{CE}(u, x)^{2} d x}
$$

Fig. 4 shows the evolution of this error if the initial data is the oblate spheroid $\left(e_{0}=0.8, e_{j}=0\right)$ for both schemes of approximation. 

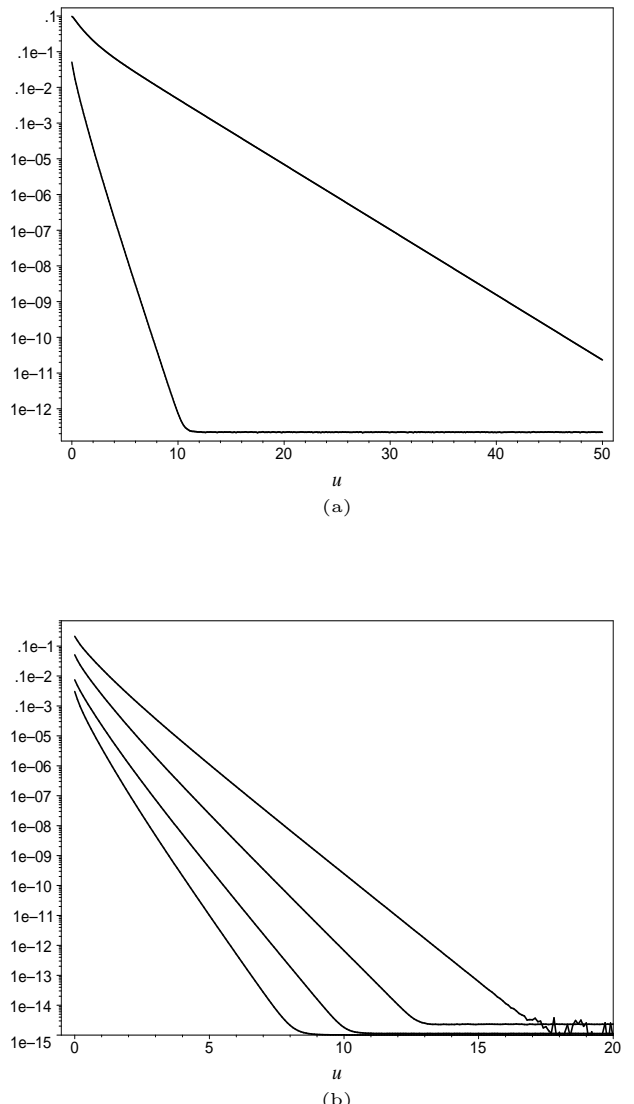

FIG. 4: (a) Evolution of the $L_{2}$ error of the constraint equation using the first and the second schemes of approximation (upper and lower curves, respectively). Here $N=7, J=2$ (see Eq. (11)) and $M=8$ collocation points, where it is clear the rapid convergence of the collocation expansion (12). In (b) we present the influence of increasing the truncation orders $N=5,7,9,11$ with respective $N+1$ collocation points. The initial conditions correspond to the oblate spheroid with eccentricity $e_{0}=0.8$.

The residual equation can provide another possible way of verifying the convergence and accuracy of the numerical method as the truncation order is increased. In fact, as demonstrated by ??, the rms error of residual equation is identified as the upper bound of the rms error between the approximate and exact solutions. Then, in our last numerical experiment the evolution of the error

$$
\sqrt{\frac{1}{2} \int_{-1}^{1} \operatorname{Res}(u, x)^{2} d x}
$$

where $\operatorname{Res}(u, x)$ is the residual equation (cf. Eq. (91)) considering only the second scheme of approximation (Eq. (12)) based on the collocation method.
In Fig. 5 the convergence is illustrated by graphs corresponding to several truncation orders, namely, $N=5,7,9,11$, and respectively $M+1$ collocation points. As it can be seem the error decreases rapidly until reaching to the value considered zero up to our numerical precision, and the greater is the truncation order less time is necessary to attain this value.

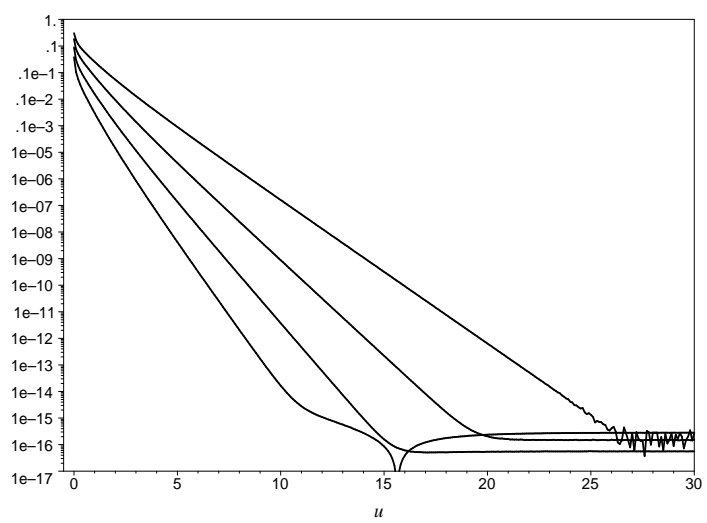

FIG. 5: Evolution of the residual error the truncation orders $N=5,7,9,11$ (upper to lower curves) and respective $N+1$ collocation points. The initial conditions correspond to the oblate spheroid with eccentricity $e_{0}=0.8$.

\section{FINAL REMARKS}

The low dynamical system approach provided by the Galerkin method constitutes a valuable tool to deal with nonlinear problems as demonstrated not only here, but also in problem of turbulence of fluids [5]. Two aspects are worth of mentioning:

- The transformation of, say, a partial differential equation to a finite set of ordinary differential equations. In general the greater is the set of these equations more accurate is the approximate solution.

- A reasonable accuracy can in fact be obtained with a relatively low dimensional dynamical system.

As a matter of fact we have applied the Gakerkin method to a problem of gravitation, namely, the dynamics of the Robinson-Trautman spacetimes that represents the simplest axisymmetric geometries endowed with gravitational waves. As we have described one of the crucial steps to implement the method is to project the residual equation into each 
basis function, which is necessary to obtain the dynamical system. Such projections are integrations in the spatial domain (in our case angular domain), but due to the presence of an exponential term, $\mathrm{e}^{-Q}$, these integrations can not be performed as desirable. Then, we have engendered two schemes to overcome this difficulty (cf. Eqs. (11) and (12)), where the decomposition of the exponential with respect to a series of Chebyshev functions using the collocation method appeared to be quite satisfactory as demonstrated by several numerical experiments. Accordingly, even for a small truncation order, say $N=7$ (and $N+1$ collocation points) to decompose $\mathrm{e}^{-Q}$, the error associated to the constraint and residual equation are acceptable. The increase of truncation error to $N=11$ - still a modest truncation order - provides a much better accuracy and fast convergence.

Finally, the combination of Galerkin and collocation methods can be very useful in studying other interesting nonlinear problems in Cosmology and Gravitation.

The authors acknowledge the financial support of the Brazilian agencies CNPq and CAPES.

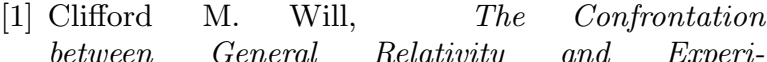
ment, Living Rev. Relativity 9, (2006), 3. http://www.livingreviews.org/lrr-2006-3

[2] Jeffrey Winicour, Characteristic Evolution and Matching, Living Rev. Relativity 8, (2005), 10. $\quad$ http://www.livingreviews.or/lrr-2005-10 Greek Cook, Initial Data for Numerical Relativity, Living Rev. in Relativity 3, (2000), 5. http://www.livingreviews.or/lrr-2005-5

[3] S. Bonazzola, E. Gourgoulhon and J. A. Marck, J. Comp. Appl. Math., 109, 433 (1999). C. Canuto , M. Y. Hussaini, A. Quarteroni and T. A. Zang, Spectral Methods in Fluid Dynamics, Springer-Verlag, Berlin, Germany, 1988.

[4] C. A. J. Fletcher, Computational Galerkin Methods, Springer-Verlag, New York Inc., 1984.

[5] P. Holmes, John. L. Lumley and Gal Berkooz, Tur- bulence, Coherent Structures Dynamical Systems and Symmetry, Cambridge University Press, Cambridge, 1998.

[6] John P. Boyd, Chebyshev and Fourier Spectral Methods, Dover Publications, Inc., 2001.

[7] I. Robinson and A. Trautman, Phys. Rev. Lett. 4, 431 (1960); Proc. Roy. Soc. A265, 463 (1962); H. P. Oliveira and I. Damião Soares, Phys. Rev. D70, 084041 (2004).

[8] E. T. Newman and T. W. J. Unti, J. Math. Phys., 3, 891 (1962).

[9] H. Bondi, M. G. J. van der Berg, and A. W. K. Metzner, Proc. R. Soc. London A269, 21 (1962); R. K. Sachs, Phys. Rev. 128, 2851 (1962).

[10] B. A. Finlayson, The Method of Weighted Residuals and Variational Principles, 1973, Academic, New York. 